Table 2. Descriptive data of MNA and NRS-2002 in rheumatic diseases.

\begin{tabular}{lccccc}
\hline \multicolumn{5}{c}{ NUTRITIONAL SCREENING TOOLS } \\
\hline & MNA & NRS-2002 & NRS-2002* & Rho & $\mathbf{p}$ \\
\hline All Rheumatic Diseases & & & & & \\
Total Score, $n(S D)$ & $24.31(3.48)$ & & & .23 & .004 \\
Malnutrition, $n(\%)$ & $4(2.6)$ & $5 \overline{(3.3)}$ & $5 \overline{(3.3)}$ & & \\
Risk, $n(\%)$ & $55(36.2)$ & $147(96.7)$ & $41(27)$ & & \\
Normal, $n(\%)$ & $93(61.2)$ & & $106(69.7)$ & & \\
Rheumatoid Arthritis & $24.43(3.23)$ & & & & \\
Total Score, $n(S D)$ & $1(1.4)$ & $2 \overline{(2.9)}$ & $2 \overline{(2.9)}$ & & \\
Malnutrition, $n(\%)$ & $22(31.4)$ & $68(97.1)$ & $15(21.4)$ & & \\
Risk, $n(\%)$ & $47(67.1)$ & & $53(75.7)$ & & \\
Normal, $n(\%)$ & & & & & \\
\hline
\end{tabular}

Conclusion: MNA was a more sensible tool for detecting risk of malnutrition when compared to NRS-2002. Screening tools play an important role at nutritional evaluation and should be complemented with objective methods such as $\mathrm{BIA} .{ }^{1}$ Rheumatologists must be aware that nutritional disorders affect the state of rheumatic diseases; and selecting an appropriate tool to detect malnutrition is of vital importance.

References:

[1] Elkan, A. C., Engvall, I. L., Tengstrand, B., Cederholm, T., \& Hafström, I. (2008). Malnutrition in women with rheumatoid arthritis is not revealed by clinical anthropometrical measurements or nutritional evaluation tools. European journal of clinical nutrition, 62(10), 1239-1247.

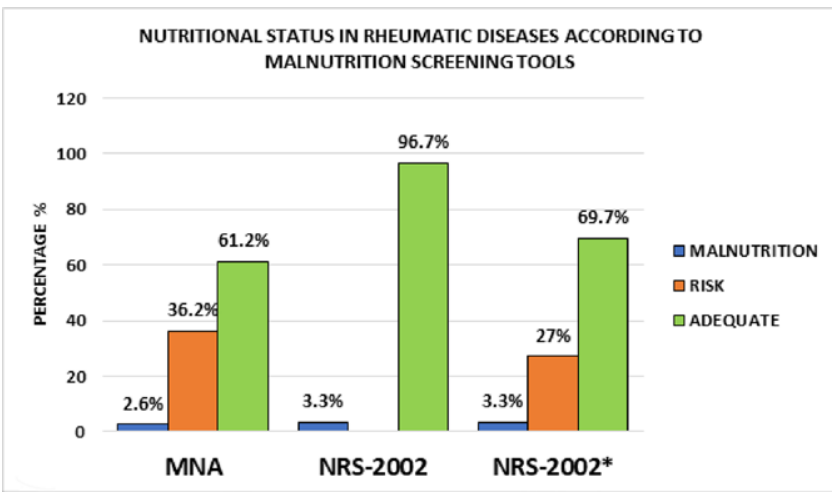

Figure 1. Graphic 1. Nutritional screening tools in rhematic disorders. Total $\mathbf{N}=152$ patients. MNA= Mini Nutritional Assessment NRS-2002= Nutritional Risk Screening Tool-2002. NRS2002* $=$ Nutritional Risk Screening Modified with 3 parameters.

Disclosure of Interests: None declared

DOI: 10.1136/annrheumdis-2020-eular.5787

\section{AB1301-HPR COMORBIDITIES AND FACTORS INFLUENCING RECURRENT GOUT ATTACK IN PATIENTS WITH GOUT: A CROSS-SECTIONAL STUDY}

Y. Jiang ${ }^{1}$, Z. Liao ${ }^{1}$, Y. Huang ${ }^{1}$, J. Liang ${ }^{2}$, Y. Xie ${ }^{1}$, J. Wu' ${ }^{1}$, J. Gu' ${ }^{1}{ }^{1}$ The Third Affiliated Hospital of Sun Yat-Sen University, Guangzhou, China; ${ }^{2} \mathrm{Huadu}$ Affiliated Hospital of Southern Medical University, People's Hospital of Huadu District, Guangzhou, China

Background: Gout attack is characterized by painful arthritis, loss of function and reduced quality of life. Frequent gout attacks can exert negative an influence on gout management [1]

Objectives: The objective was to identify the comorbidities of gout, to compare gender difference and to identify independent factors of multiple gout attacks. Methods: A cross-sectional study was performed to collect demographic, clinical variables, self-reported comorbidities, and relevant testing. Group comparison and correlation of serum uric acid ( $\mathrm{SUA}$ ) levels with other variables was performed. Univariate and multivariate logistic regression was used to detect independent risk factors of sUA.

Results: 653 gout patients were enrolled, including $553(84.7 \%)$ males. The mean age was $48.3 \pm 15.8$ years old, with a disease duration of $8.0 \pm 6.4$ years. 170 (26.0\%) patients had hypertension, and $57(8.7 \%)$ had hyperlipidemia. Elevated total cholesterol (TC) was observed in $173(26.5 \%)$ cases. $42(37.1 \%)$ cases presented with increased triglycerides (TG) and $270(41.3 \%)$ had increased low-density lipoprotein (LDL-C). Abnormalities including nephrolithiasis (29.4\%), hydronephrosis $(3.2 \%)$, and gallstones $(11.9 \%)$ were detected in the patients who underwent ultrasound examination. Although female patients had a longer disease duration, they had lower levels of sUA, creatine and C-reactive protein (CRP). A positive correlation with SUA was found in TG and CRP $(P<0.05)$ in female patients, which was not observed in males. Only gout duration (OR=1.406, $P<0.001)$, sUA (OR=1.006, $P<0.001)$ and LDL-C $(\mathrm{OR}=0.530, P=0.006)$ were independent factors of gout attack (>20 times).

Conclusion: Comorbidity screening involving dyslipidemia is often neglected in gout patients. Gout duration and SUA level are risk factors of multiple gout attacks.

References:

[1] Wang Y, Yan S, Li C et al. Risk Factors for Gout Developed From Hyperuricemia in China: A Five-Year Prospective Cohort Study. Rheumatol Int. 2013; 3 705-10.

Table 1 Comparisons of clinical and laboratory variables between male and female patients with gout

\begin{tabular}{|c|c|c|c|}
\hline Variable & Male $(n=553)$ & Female $(n=100)$ & $p$ \\
\hline Age (years) & $47.7 \pm 15.9$ & $51.3 \pm 14.5$ & $0.040^{*}$ \\
\hline BMI (kg/ m2) & $24.5(22.0-27.0)$ & $23.3(20.3-25.3)$ & $0.001^{*}$ \\
\hline Gout duration (years) & $6.0(3.0-11.0)$ & $9.5(4.0-15.0)$ & $0.001^{*}$ \\
\hline \multicolumn{4}{|l|}{ Gout attack times } \\
\hline$\bullet \leq 5$ & $209(37.5)$ & $33(33.0)$ & \\
\hline$\cdot 6-10$ & $97(17.5)$ & $22(22.0)$ & \\
\hline$\bullet 11-20$ & 70 (12.7) & $22(23.0)$ & \\
\hline$\bullet>20$ & $177(32.0)$ & $23(23.0)$ & \\
\hline \multicolumn{4}{|l|}{ Comorbidies } \\
\hline Hypertension, n (\%) & $138(25.0)$ & $32(32.0)$ & 0.140 \\
\hline Coronary heart disease, $\mathrm{n}(\%)$ & $57(10.3)$ & $10(10.0)$ & 0.926 \\
\hline Fatty liver disease & $53(9.6)$ & $4(4.0)$ & 0.069 \\
\hline \multicolumn{4}{|l|}{ Laboratory testing } \\
\hline -ALT(U/L) & $25.0(17.0-40.0)$ & $19.2(14.6-29.0)$ & $0.003^{*}$ \\
\hline -ALB(g/L) & $43.5 \pm 6.3$ & $42.4 \pm 3.9$ & $0.025^{\star}$ \\
\hline •TB $(\mu \mathrm{mol} / \mathrm{L})$ & $12.7(9.0-17.3)$ & $14.5(12.1-17.4)$ & $0.002^{*}$ \\
\hline •BUN (mmol/L) & $4.85(3.98-6.27)$ & 4.85 (3.91-4.82) & 0.588 \\
\hline -Creatine (mmol/L) & $94.0(81.4-108.1)$ & $73.8(67.4-87.2)$ & $<0.001^{*}$ \\
\hline -UA $(\mu \mathrm{mol} / \mathrm{L})$ & $528.4 \pm 141.1$ & $363.8 \pm 122.9$ & $<0.001^{*}$ \\
\hline •Glucose (mmol/L) & $5.62 \pm 1.67$ & $5.43 \pm 1.17$ & 0.317 \\
\hline •TC (mmol/L) & $5.05 \pm 1.15$ & $5.09 \pm 1.10$ & 0.726 \\
\hline •TG (mmol/L) & $2.11 \pm 1.66$ & $1.94 \pm 1.86$ & 0.335 \\
\hline •HDL-C (mmol/L) & $1.14 \pm 0.30$ & $1.52 \pm 0.41$ & $<0.001^{*}$ \\
\hline -LDL-C (mmol/L) & $3.25 \pm 0.97$ & $3.39 \pm 0.92$ & 0.182 \\
\hline •ESR $(\mathrm{mm} / \mathrm{h})$ & $40.1 \pm 32.4$ & $37.4 \pm 20.8$ & 0.402 \\
\hline -CRP (mg/L) & $28.0 \pm 40.3$ & $10.5 \pm 18.6$ & $<0.001^{*}$ \\
\hline
\end{tabular}

* $p<0.05$; BMI: body mass index; sUA: serum uric acid; ALT: alanine aminotransferase; ALB: albumin; TB: Total bilirubin; BUN: blood urea nitrogen; UA: uric acid; TC: total cholesterol; TG: triglycerides; HDL-C: high-density lipoprotein; LDL-C: Low-density lipoprotein; ESR: erythrocyte sedimentation rate; CRP: C-reactive protein

Acknowledgments: None

Disclosure of Interests: None declared

DOI: 10.1136/annrheumdis-2020-eular.728

\section{AB1302-HPR ASSOCIATIONS OF SLEEP ON GOUT PREVALENCE AND SERUM URIC ACID CONCENTRATIONS}

P. Kachur ${ }^{1}$, S. Tadepalli ${ }^{2}$, C. Pramil ${ }^{2} .{ }^{1}$ Ochsner Medical Center, New Orleans, United States of America; ${ }^{2}$ Ocean Medical Center, Brick, United States of America

Background: Sleep deprivation and sleep inconsistency have been associated with modulation of the immune and inflammatory response systems with effects that include higher risk for psychosis, cardiovascular disease, and increased susceptibility to infection. One hypothesized mechanism to explain these findings is an association with pro-inflammatory states.

Objectives: In a first-of-its kind study, we examined associations between sleep quantity and gout using the largest public US dataset available; the National Health and Nutrition Examination Survey (NHANES).

Methods: NHANES data from 2009 to 2014 was selected based on questionnaire responses and laboratory studies. The resulting data set was analyzed fo serum uric acid (SUA) levels (cut-off $>6.0 \mathrm{mg} / \mathrm{dL}$ in females and $>7.0 \mathrm{mg} / \mathrm{dL}$ in males), and gout risk factors. Analysis of variance, means, proportions, as well as univariate and multiple logistic regression models were used to evaluate the associations between sleep quantity, SUA and gout.

Results: 10,410 survey participants from 2003 to 2014 were included in the study. A total of $280(2.7 \%)$ had gout. Gout prevalence ranged between $4.0 \%$ and $4.7 \%$ between high, normal, and low sleep groups. Multivariate analyses showed a significant association between extremely low sleep levels $(\mathrm{OR}=0.53$ and $\mathrm{Cl}$ $=0.34$ to 0.83 ), and gout, which was also seen with SUA level association (OR $=0.41$ and $\mathrm{Cl}=0.32$ to 0.52 ). However, in the broader sense, lower than normal ( $<7$ hours) and higher than normal ( $>9$ hours) levels of sleep had no significant associations with either gout prevalence or SUA, likely due to a non-linear relationship of SUA to sleep levels.

Conclusion: Despite numerous adverse effects found with low sleep levels, our study does not show a strong association between sleep quantity and gout prevalence or SUA levels.

References: 
[1] Hasday et al. "Nocturnal increase of urinary uric acid: creatinine ration. A biological correlate of sleep-associated hypoxemia." AM Rev Respir Dis 1981, 135:534

[2] Lavie, Lena. "Obstructive sleep apnoea syndrome-an oxidative stress disorder" Sleep Medicine Reviews 2003, 7:35

[3] Roddy et al. "The Association of Gout with Sleep Disorders: a cross-sectional study in primary care."BMC Musculoskeletal Disorders 2013, 14:119

[4] Sahebjani $\mathrm{H}$. "Changes in urinary uric acid excretion in obstructive sleep apean before and after therapy with nasal continuous positive airway pressure." Chest 1998' 113: 1604

[5] Taheri et al. "Short Sleep Duration is Associated with Reduced Leptin, Elevated Ghrelin, and Increased Body Mass Index" PLOS Medicine 2004, $1: 210$

Disclosure of Interests: None declared

DOI: 10.1136/annrheumdis-2020-eular.1369

\section{AB1303-HPR $\quad$ TIME UNTIL DIAGNOSIS IN RHEUMATOLOGICAL PRACTICE: RESULTS FROM A CROSS-SECTIONAL MIDDLE-EUROPEAN COHORT COMPARED TO DATA FROM A SYSTEMATIC LITERATURE REVIEW}

R. Mc-Cutchan ${ }^{1}$, S. Maier ${ }^{1}$, V. Winkler ${ }^{1}$, B. Gruber ${ }^{1}$, M. Schirmer ${ }^{1}$ on behalf of Rheumatology working group of diploma students, Innsbruck. ${ }^{1}$ Medizinische Universität Innsbruck, Innsbruck, Austria

Background: The time from first symptom to diagnosis (= diagnostic delay) is considered as key factor for better outcome in many chronic inflammatory rheumatic diseases, especially for rheumatoid arthritis (RA) and vasculitides like giant cell arteritis (GCA). A longer diagnostic delay may cause pain, reduced functionality, reduced life-quality and increased morbidity, as well as structural damages of the organs linked with higher mortality. This retrospective study assessed the diagnostic delay in consecutive Middle-European outpatients and compared results with those of a systematic literature review (SLR).

Objectives: To compare disease-specific diagnostic delays of consecutive rheumatic patients with international data from a systematic literature review.

Methods: Charts of a single-centre cohort with consecutively recruited patients were retrospectively reviewed for patients' and diseases' characteristics at a Middle-European university outpatient clinic for rheumatology. A SLR was performed according to PRISMA guidelines.

Results: The average mean \pm SD time from first symptom to established diagnosis was $7.9 \pm 11.7(0.02-56.7)$ years. Spondyloarthritis patients showed the longest diagnostic delay with $13.1 \pm 14.2(0.1-56.7)$ years, whereas polymyalgia rheumatica-patients had the shortest diagnostic delay with $1.5 \pm 0.4$ (0.3-18.0) months. In the SLR, most data for diagnostic delays are comparable to the Innsbruck cohort, but the diagnostic delay for psoriatic arthritis in Innsbruck is longer than in the Danish DANBIO registry $(p<0.001)$. Independent risk factors for prolonged diagnostic delays could not be identified.

Conclusion: For this Middle-European area, initiatives are justified especially to shorten diagnostic delays of SpA and PsA.

References:

[1] O'Dell JR. Treating rheumatoid arthritis early: A window of opportunity? Arthritis Rheum. 2002;46:283-5.

[2] Seo MR, Baek HL, Yoon HH, Ryu HJ, Choi HJ, Baek HJ, et al. Delayed diagnosis is linked to worse outcomes and unfavourable treatment responses in patients with axial spondyloarthritis. Clin Rheumatol. 2015;34:1397-405.

[3] Diagnostic delay of more than 6 months contributes to poor radiographic and functional outcome in psoriatic arthritis. Ann Rheum Dis. 2015;74:1045-50.

[4] Dejaco C, Brouwer E, Mason JC, Buttgereit F, Matteson EL, Dasgupta B. Giant cell arteritis and polymyalgia rheumatica: current challenges and opportunities. Nat Rev Rheumatol. Nature Publishing Group; 2017;13:578-92.

Acknowledgments: We acknowledge and thank all patients who could be recruited to the SolutionX project. Ethical vote was obtained by the local ethics committee of the Medical University of Innsbruck (AN2017-0041 370/4.18).

Disclosure of Interests: Rick Mc-Cutchan: None declared, Sarah Maier: None declared, Valentin Winkler: None declared, Bernhard Gruber: None declared, Michael Schirmer Grant/research support from: total <3000.- $€$, Speakers bureau: total $<3000$. $€$

DOI: 10.1136/annrheumdis-2020-eular.3724

\section{AB1304-HPR KNOWLEDGE OF HIGH SCHOOL STUDENTS ABOUT RHEUMATIC DISEASES: EFFECT OF AN EDUCATIONAL SESSION JOINTLY PROVIDED BY PORTUGUESE EULAR ASSOCIATIONS}

G. Pimentel ${ }^{1}$, A. Marques ${ }^{2,3}$, R. Ferreira ${ }^{2,3}$, E. Mateus ${ }^{4}$, A. Pais ${ }^{5} .{ }^{1} \mathrm{CHUC}$, Internal Medicine, Coimbra, Portugal; ${ }^{2} \mathrm{CHUC}$, Rheumatology, Coimbra, Portugal; ${ }^{3}$ UICISA:E, ESEnfc, Coimbra, Portugal; ${ }^{4}$ Portuguese League Against
Rheumatic Diseases, Lisboa, Portugal; ${ }^{5}$ Portuguese Association of Childhood with Arthritis and Rheumatism, Lisboa, Portugal

Background: International studies have shown that the awareness and knowledge of the general population about rheumatic and musculoskeletal diseases (RMDs) is poor. This is even lower in the young population, which are also affected by these disease but do not have as much awareness campaigns as adult community. Their professors and primary health care professionals may also play here also a key role, promoting early detection of signs and interpretations of symptoms, thus avoiding late health care referrals and diagnosis. (Vlieland, 2016).

Objectives: To assess the knowledge of high school Portuguese students about the RMDs and raise awareness for RMDs in young people, their professors, vig ilants, and primary care nurses, within the school environment.

Methods: A 1-hour educational session about RMDs was planned (with the inputs from members of the Portuguese EULAR Associations) and performed during school activities. The educational session started with a knowledge questionnaire about RMDs in a paper sheet (9 questions; Graph 1), repeated in the end. An interactive session, using slides, interactive questions (Sli.do®), and practical demonstrations to simulate RMD symptoms (e.g. stiffness and functional limitations) was then lead by a rheumatology nurse, with the testimony from a young patient representative. A primary care nurse assisted in order to be engaged and promote future sessions ("autonomously"). Change in knowledge was assessed with Wilcoxon-test and awareness was documented with "word clouds" (using Sli.do®).

Results: A total of 75 students participated in four sessions (mode=16 years). Half of students $(52 \%)$ had never heard about RMDs. Knowledge increased significantly in all questions ( $p<0.001$; Graph 1). Figures 1 and 2 document the most common words representing what defines a RMD and what are the main symptoms, respectively.

Graph 1. Change in the percentage of correct answers before and after na educational session $(n=75$ ) (all $p<0.001$ )

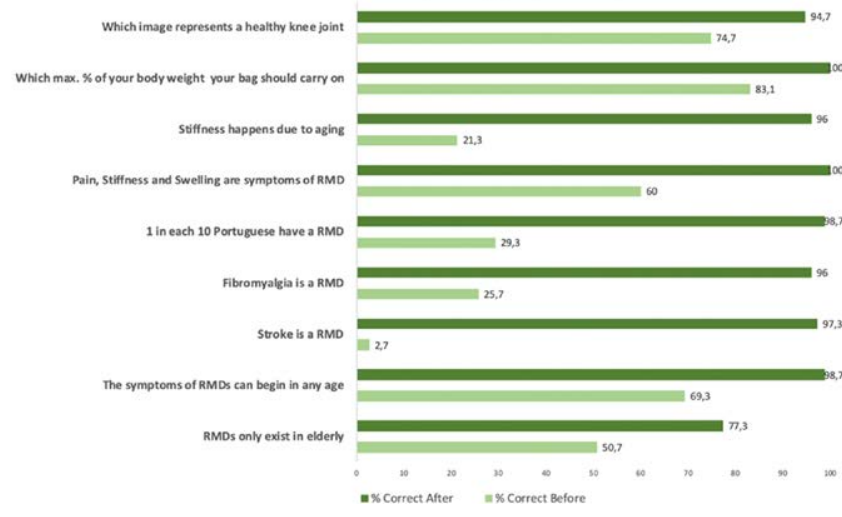

Figure 1. "One to two words" to define "Rheumatic diseases" by college students $(n=75)$ after an educational session

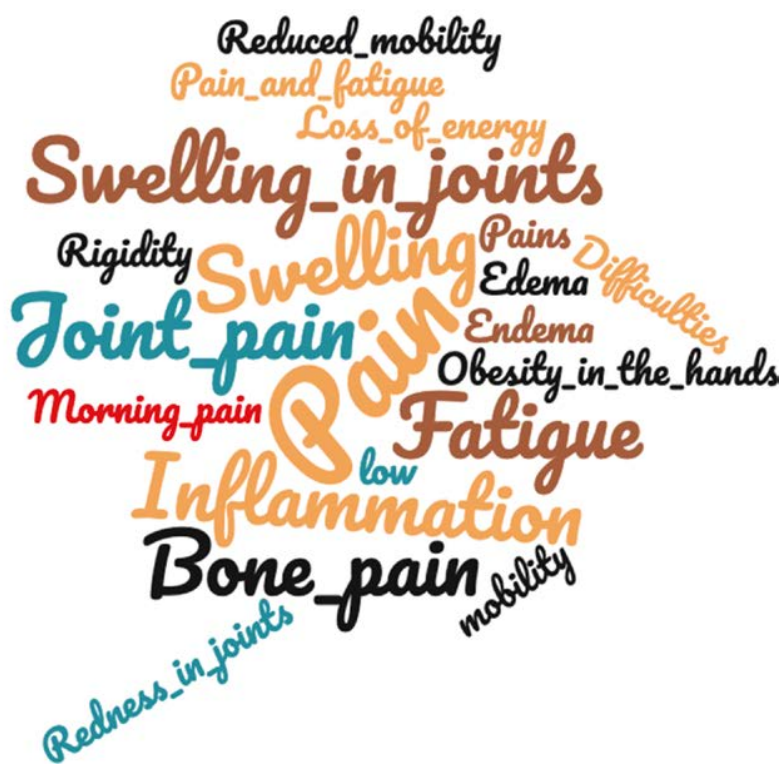

